\title{
Causas principales de muerte entre 2.708 heroinómanos del noreste italiano (1985-1998)
}

\author{
Quaglio, G. '; Talamini, G. ${ }^{2}$; Lugoboni, F.'; Lechi, A. ${ }^{2}$; Venturini, L. '; GiCS ${ }^{3}$; Mezzelani, P. ${ }^{1}$ \\ (1) Servizio di Medicina delle Dipendenze, Istituto di Medicina Interna C, Universita di Verona. \\ (2) Istituto di Medicina Interna C, Università di Verona. \\ (3) Gruppo Intersert di Collaborazione Scientifica (G.I.C.S.).
}

Enviar correspondencia a:

Prof. Paolo Mezzelani, Servizio di Medicina delle Dipendenze, Policlinico GB Rossi, Università di Verona, 37134 Verona. Tel: 0458074604 Fax: 0458074269 email: paolo.mezzelani@univr.it

\section{RESUMEN}

Objetivos. Establecer las causas de la muerte acaecidas entre 1985 y 1998 en un numeroso grupo de usuarios de droga por vía intravenosa (ADVP)] acogidos en 36 Servicios Públicos para Toxicomanías (SerT) del noroeste italiano.

Métodos. Estudio realizado sobre datos recogidos en los archivos de los Ser. Ty del Registro Municipal de cada una de las ULSS estudiadas.

Individuos implicados. Todos los ADVP que hayan pasado por lo menos una vez por uno de los 36 Ser.T. incluidos en el estudio.

Resultados. El estudio analizó las causas de la muerte de 2708 individuos. La primera causa de muerte es la sobredosis (37\%), seguida del SIDA (32.5\%) y de los accidentes de tráfico (9.4\%). El porcentaje de muertes causadas por el SIDA aumentó progresivamente, pasando de un $2.7 \%$ en 1985 a un $42.2 \%$ en 1996, reduciéndose después al $16.9 \%$ en 1998. El porcentaje de fallecimientos por sobredosis se mantuvo prácticamente constante durante todo el tiempo. La edad media de la muerte aumentó, pasando de 26 años a mediados de los años 80 a 34 años en 1998. La tasa de mortalidad entre ADVP es 13 veces mayor que en el conjunto de la población (95\%, Cl 11.3-14.6). En el conjunto de la población el porcentaje de fallecimientos en edades comprendidas entre los 15 y los 34 años atribuida al uso de opiáceos era en 1991 (año del último censo) del 16\%. La prevalencia del VIH no resultó estadísticamente significativa en las causas de muerte por suicidio o sobredosis.

Conclusiones. Se comprobó que la tasa de mortalidad era 13 veces superior a la de la población general. Se demostró la importancia del sexo femenino y el drop out de los tratamientos (al margen del tipo de tratamiento en cuestión) como factores de riesgo de sobredosis. La paulatina disminución de muertes por SIDA destaca la importancia de los tratamientos preventivos y terapéuticos adecuados contra la infección de VHC. Las medidas que se adopten en el campo de las drogodependencias, tanto en el ámbito de la política como en la investigación y la formación, deben tener presente el dramáticamente elevado porcentaje de individuos que mueren por consumo de heroína.

Palabras clave: heroinómanos, mortalidad, Sida, sobredosis, estudio retrospectivo.

\section{ABSTRACT}

Aims: To ascertain the causes of deaths among a very large cohort of heroin injecting drug users (IDUs) who, from 1985 to 1998, attended 36 Public Health Authority Centres for Drug Users (PCDUs) in north-eastern Italy.

Design: Retrospective analysis of data, obtained from the Annual Register of each Centre and the Municipal Registry Office of each local health district.

Setting: Thirty-six PCDUs in north-eastern Italy and Medical Service for Addictive Disorders of the University of Verona.

Participants: All IDUs who had sought medical care at least once in the PCDUs during the study period.

Findings: Of 2708 deaths, overdose was found to be the major cause $(37 \%)$, followed by AIDS $(32.5 \%)$ and road accidents $(9.4 \%)$. The percentage of deaths due to AIDS increased steadily from $2.7 \%$ in 1985 to $42.2 \%$ in 1996, and then decreased to $16.9 \%$ in 1998 . Deaths due to overdose remained almost constant. The average age of death per year rose from 26 in the mid eighties to 34 in 1998. The mortality rate among IDUs proved much higher compared to the general population of the same age (13-fold, $95 \% \mathrm{Cl} 11.3-14.6)$. The proportion of all deaths attributable to regular use of illegal opiates in the 15-34 age group in the general population in 1991 was $16 \%$. HIV prevalence was not a significant factor in suicides and deaths by overdose.

Conclusions: The mortality rate was 13 times greater than in the general population. To be female and to have dropped out of any kind of treatment proved an important risk factor for overdose. The fall in deaths from AIDS enhances the problem to prevent and treat HCV infection. Decisions in drug projects, in research and in training should be influenced by the strikingly high percentage of deaths due to drug use.

Key words: heroin addict, mortality, Aids, overdose, retrospective analysis. 


\section{INTRODUCCIÓN}

E n los usuarios de drogas por vía intravenosa (ADVP) la tasa de mortalidad supera con mucho la de la población general de la misma edad y el mismo sexo ${ }^{1-4}$. La aparición del SIDA contribuyó en gran medida al incremento de la mortalidad entre ADVP ${ }^{5-8}$.

La mayoría de los estudios sobre relaciones entre drogodependencia de opiáceos y mortalidad se realizan sobre grupos de individuos numéricamente limitados, y por ello muchas de las estimaciones del aumento de la mortalidad por uso ilegal de opiáceos no están avaladas científicamente. Por otra parte, las dificultades para obtener información sobre las causas de la muerte, sumadas a la falta de datos concretos sobre la población de base y, especialmente, sobre el número de ADVP, evidencian los problemas que tienen que afrontar este tipo de estudios y, de cualquier modo, lo complicado que resulta conseguir resultados fiables.

El presente trabajo se ha realizado en base a un número de muertes que supera en más del doble las recogidas en otra investigación que habíamos efectuado previamente $^{13}$. Se llevó a cabo en las regiones del Véneto y Trentino-Alto-Adige, incorporando 10 Ser.T. (Servicios públicos para Toxicomanías) nuevos e implicando a un total de 36 Servicios. El periodo de observación del presente trabajo se extiende desde el 1985 al 1998.

Los Ser.T. implicados forman parte del Gruppo Intersert di Collaboraziones Scientifica (GICS). Fundado en 1985, el GICS sirvió de plataforma de encuentro entre el servicio de Medicina de las Dipendenze dell'Azienda Ospedaliera [Secciones de la Administración Hospitalaria] - Università di Verona y los Servicios territoriales para las drogodependencias.

En Italia, con una población de alrededor de 58.000.000 de habitantes, existen 569 Ser.T., una media de uno por cada 100.000 personas.

El número de usuarios por Servicio varía mucho, pudiendo oscilar entre menos de 100 y más de mil.

Como es sabido, los Ser.T. intervienen en ámbitos muy diversos de la drogodependencia, siendo éste un tema muy extenso que no podemos abordar en las páginas que siguen. ${ }^{14}$

El Véneto y el Trentino-Alto-Adige son regiones de un elevado nivel de industrialización con una densidad media de población de 170 habitantes por Km. cuadrado, ${ }^{15}$ una tasa de desempleo del $3.95 \%$, una tasa de analfabetismo muy pequeña que sólo afecta a la población anciana; el 4.9\% de la población es licenciado; ${ }^{16}$ la renta per capita media por habitante en 1996 era de 19.000 euros (la renta per capita media italiana en el mismo año fue de 15.300 euros). ${ }^{17}$

\section{METODOS}

Población estudiada. El estudio fue realizado en base a los datos obtenidos sobre una población de
4.960.000 habitantes. Se incluyeron todos los pacientes heroinómanos, con posible uso concurrente de otras drogas, que hubiesen estado una vez por lo menos en el Ser.T. entre 1985 y 1998; la suma total ascendió a 33.072 individuos. Los 2708 fallecimientos pertenecen sólo a este grupo de consumidores.

Para comparar la tasa de mortalidad de los ADVP con la de la población general acudimos a los datos que del último censo italiano de 1991, calculando la tasa de mortalidad estimada por sexos, y dividiendo las edades en grupos de 5 años.

Obtención de los datos. Las causas de la mortalidad se establecieron consultando el archivo de los Ser.T. y el Registro Municipal de cada una de las ULSS implicadas. También se recogieron datos de los historiales clínicos de los Servicios, y cuando fue posible, de los historiales clínicos correspondiente a estancias eventuales (esto último se pudo realizar en un 20\% de los casos) y, muy raramente, de informes de autopsias.

Las causas de la muerte se dividieron en 7 categorías: sobredosis, SIDA, accidentes de tráfico, suicidio, hepatopatías (cirrosis y hepatocarcinoma), otras (causas fortuitas, homicidio, neoplasias malignas, patologías cerebrovasculares, cardiopatías, enfermedades infecciosas y otras enfermedades) y causas desconocidas.

A diferencia de otros estudios, y teniendo en cuenta su elevado número, en el nuestro los accidentes de tráfico se consideraron una categoría independiente; esto ha permitido establecer una valoración autónoma respecto a otras causas de muerte violenta.

Siguiendo la definición de van Haastrecht et al. ${ }^{18}$ hemos considerado muertes fortuitas por sobredosis los casos de uso de droga evidente y reciente en circunstancias que excluirían la posibilidad de otras causas (presencia de droga, jeringuillas, banda hemostática, limón o ácido cítrico, algodón, etc. en las cercanías del cadáver), y muertes por suicidio aquellas en las que se encontraban sujetos fallecidos por muerte auto infligida o planificada (acompañada de notas de despedida, por ejemplo).

Los pacientes con SIDA se consideraron muertos por SIDA tras descartar la posibilidad de otras causas. Siguiendo la definición de Galli y Musicco ${ }^{5}$ la causa de muerte se consideró desconocida en los casos en que los datos disponibles eran insuficientes para establecer exactamente su origen.

Análisis estadístico. Para establecer las diferencias entre los distintos grupos se usaron el Chi cuadrado y el test de ANOVA. Para las comparaciones múltiples se aplicó el índice de Bonferroni.

Se comparó la tasa de los ADVP con la del conjunto de la población estudiada del Véneto y del Trentino Alto Adige $^{19,20}$. Después de corregir la información sobre edad y sexo con los datos del último censo, la relación entre muertes observadas/muertes estimadas (O/E) equivale a la Standardised Mortality Ratio (SMR). 
Para confrontar los hechos observados con los estimados ${ }^{21}$, se usaron test asumiendo que la distribución de los casos de muerte observados es de Poisson. Para calcular los límites de confianza de la SMR ${ }^{21}$ se usó la aproximación de Byar (considerándose significativo un $\mathrm{p}<0.05)$. Los datos recogidos se analizaron con el programa estadístico SPSS (SPSS Inc., Chicago, IL).

\section{RESULTADOS}

Causas de muerte. Hemos valorado las causas de muerte de 2708 individuos (Tabla 1): 2270 (84\%) hombres y 438 (16\%) mujeres, con una relación hombres/mujeres de 5.25:1, que refleja la relación hombres/mujeres de los ADVP registrados en los Servicios del noreste de Italia (5:1).

Tabla 1. Causas de muerte diferenciadas por sexos de los 2.708 heroinómanos

\begin{tabular}{|llll|}
\hline Causas & M (\%) & F (\%) & Total (\%) \\
\hline Sobredosis & $876(38.6)$ & $125(28.5)^{\dagger}$ & $1001(36.9)$ \\
SIDA & $702(30.9)$ & $180(41.1)^{\dagger}$ & $882(32.5)$ \\
Accidente tráfico & $221(9.7)$ & $35(8)$ & $256(9.4)$ \\
Suicidio & $129(5.7)$ & $22(5)$ & $151(5.5)$ \\
Enfermedad Hepática & $83(3.7)$ & $14(3.2)$ & $97(3.5)$ \\
Otras causas & $106(4.7)$ & $30(6.8)$ & $136(5)$ \\
Sin datos & $153(6.7)$ & $32(7.3)$ & $185(6.8)$ \\
Totales $(\%)$ & $2270(84)^{*}$ & $438(16)^{*}$ & 2708 \\
\hline${ }^{\dagger}=$ Varones vs hembras p<0.001; ${ }^{*}=$ Porcentajes por fila & & \\
\hline
\end{tabular}

La primera causa de muerte es la sobredosis (37\%), seguida del SIDA (32.5\%), accidentes de tráfico $(9.4 \%)$, suicidio $(5.5 \%)$, y hepatopatías $(3.5 \%)$; las causas desconocidas constituyen cerca de un $6.8 \%$, y las otras causas, alrededor del $5 \%$.

En los hombres la sobredosis es la primera causa de muerte (38.6\%), seguida del SIDA (31\%); mientras que en las mujeres la causa de muerte más importante es el SIDA (41\%), seguida de la sobredosis (28.5\%). La comparación entre las causas de muerte y sexo se considera estadísticamente significativa $(p<0.001)$.
Hasta 1991 la primera causa de muerte fue la sobredosis, seguida del SIDA (Fig. 1); entre 1992 y fines de 1996, el SIDA se convirtió en la principal causa de muerte, seguida de la sobredosis, que volvió a convertirse en principal causa de muerte desde 1997 en adelante. La tercera causa de muerte - que se mantuvo constante durante los 14 años de observación - fueron los accidentes de tráfico.

El porcentaje de muertes ocasionadas por el SIDA aumentó constantemente desde un $2.7 \%$ en 1985 al $42.2 \%$ en $1996(p<0.001)$, pasando después a disminuir drásticamente a un $16.9 \%$ en 1998, mientras que

\section{Figura 1. Causas de muerte entre 2.708 ADVP y porcentajes relativos del 1985 al 1998}

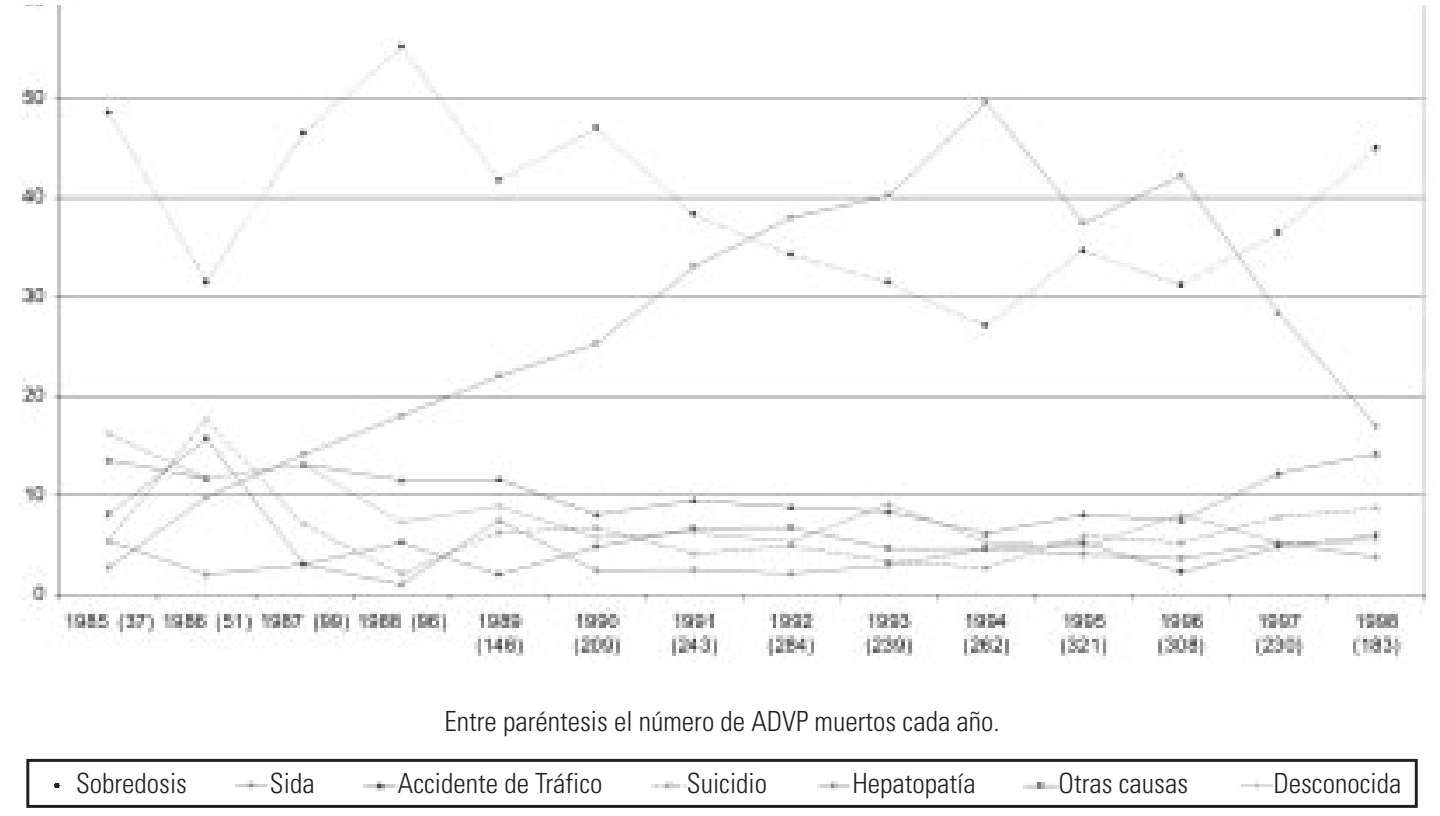


las muertes debidas a sobredosis, tras crecer continuamente hasta 1990, se mantuvieron constantes. La evolución de las causas de muerte por sobredosis y SIDA resultó estadísticamente significativa $(p<0.001)$ durante todo el tiempo que duró la observación. Sin embargo, en las otras causas de muerte no se pudieron demostrar cambios significativos.

En la categoría otras causas de muerte (Tabla 2) que supone el $5 \%$ del total, las causas de muerte fueron, de mayor a menor: homicidio (1.2\%), enfermedades cardiovasculares $(1.2 \%)$, cáncer $(1 \%)$, accidentes $(0.7 \%)$, enfermedades infecciosas $(0.5 \%)$, otras enfermedades $(0.4 \%)$ y enfermedades cerebrovasculares $(0.1 \%)$.

Causas de muerte por grupos de edad. Las principales causas de muerte clasificadas por grupos de edad aparecen en la Tabla 3, apareciendo diferencias significativas $(p<0.001)$. La sobredosis es la primera causa de muerte entre los más jóvenes (15-24 años), seguida de los accidentes de tráfico y el suicidio. En el grupo de edad entre 25-29 años, la sobredosis sigue siendo la principal causa de muerte, seguida del SIDA y de los accidentes de tráfico. Después de los 30 años el SIDA se convierte en la primera causa de muerte, seguida de la sobredosis.

\section{Tabla 2. Otras causas de muerte}

\begin{tabular}{|lrr|}
\hline Causa & No. & $\mathbf{( \% )}$ \\
\hline Accidente & 18 & $\mathbf{( 0 . 7 )}$ \\
Homicidio & 32 & $\mathbf{( 1 . 2 )}$ \\
Neoplasia & 25 & $\mathbf{( 1 )}$ \\
Hematopoiético o linfático & 10 & $(0.4)$ \\
Otras formas de neoplasia & 15 & $(0.6)$ \\
Enfermedades cerebrovasculares & 4 & $\mathbf{( 0 . 1 )}$ \\
Enfermedades cardíacas & 32 & $\mathbf{( 1 . 2 )}$ \\
Infarto miocardio & 10 & $(0.4)$ \\
Otras enfermedades cardíacas & 22 & $(0.8)$ \\
Enfermedades infecciosas & 15 & $\mathbf{( 0 . 5 )}$ \\
Endocarditis & 5 & $(0.2)$ \\
Pulmonía & 4 & $(0.1)$ \\
Septicemia & 4 & $(0.1)$ \\
Otras enfermedades infecciosas & 2 & $(0.1)$ \\
Otras enfermedades & 12 & $\mathbf{( 0 . 4 )}$ \\
TOTAL & 138 & $\mathbf{5 \%}$ \\
\hline
\end{tabular}

Tabla 3. Causas de muerte diferenciadas por edad de los 2.708 heroinómanos

\begin{tabular}{|ccccccccc|}
\hline Edad & Sobredosis & SIDA & Suicidio & $\begin{array}{c}\text { Accidente } \\
\text { tráfico }\end{array}$ & $\begin{array}{c}\text { Enfermedad } \\
\text { hepática }\end{array}$ & $\begin{array}{c}\text { Otras } \\
\text { causas }\end{array}$ & Sin datos & Total (\%) \\
\hline $15-19$ & 22 & 0 & 4 & 7 & 2 & 2 & 1 & $38(1.4)$ \\
$20-24$ & 201 & 30 & 33 & 73 & 7 & 14 & 27 & $385(14.2)$ \\
$25-29$ & 330 & 177 & 45 & 83 & 13 & 32 & 65 & $745(27.5)$ \\
$30-34$ & 256 & 378 & 30 & 68 & 42 & 48 & 47 & $869(32.1)$ \\
$35-39$ & 141 & 223 & 27 & 18 & 20 & 20 & 31 & $480(17.7)$ \\
$40-59$ & 51 & 74 & 12 & 7 & 13 & 20 & 14 & $191(7.1)$ \\
\hline
\end{tabular}

Estatus del VIH. En el conjunto de los 2708 sujetos examinados, 1254 (46.3\%) eran positivos al VIH (80\% hombres, 20\% mujeres), 876 (32.3\%) negativos al VIH (87\% hombres, 13\% mujeres); en 578 (21.4\%) casos, el estado serológico era desconocido (Tabla 4). Entre los pacientes muertos por enfermedades hepáticas hubo un $51.5 \%$ positivos al VIH, un porcentaje significativamente más alto que el de las muertes debidas a otras causas, exceptuando a los muertos por SIDA ( $p<0.001)$. En los ADVP fallecidos por sobredosis y suicidio no se observó un aumento significativo de seropositividad al VIH.

Edad, duración de la drogodependencia y duración de la acogida en el SerT. La edad media de los fallecimientos es de 31.3 años (hombres 31.3, mujeres 31.2) (Tabla 5). Los sujetos muertos por SIDA y por enfermedades hepáticas eran significativamente más viejos que los muertos por las causas recogidas en otras categorías (33.5 y 33.4 respectivamente)
( $p<0.001)$; los ADVP fallecidos por accidentes de tráfico o por sobredosis (28.3 y 29.9 respectivamente) eran considerablemente más jóvenes que los muertos por otras categorías $(p<0.001)$.

La duración de la drogodependencia pudo establecerse en 2432 casos (90\%), quedando 276 casos en los que no se pudo conocer. Se calculó una duración media de la drogodependencia de 11.7 años, considerándose similar en ambos sexos. Los pacientes muertos por SIDA y por enfermedades hepáticas tienen un historial de dependencia más prolongado (14.3 y 13.9 años respectivamente) que los fallecidos por otras categorías $(p<0.001)$; los periodos más cortos de drogodependencia aparecen entre los ADVP fallecidos por accidente de tráfico y suicidio (8.5 y 8.2 años respectivamente) $(p<0.001)$.

El periodo de acogida en el Servicio era conocido en 2668 casos (98\%) y desconocido en 40 casos. La duración media de la acogida es de 6.8 años. Los 
Tabla 4. Serología por HIV en los 2.708 ADVP

\begin{tabular}{|lccc|}
\hline Causa de la muerte & HIV + (\%) & HIV - (\%) & HIV sin datos (\%) \\
\hline Sobredosis & $184(14.7)$ & $508(58)$ & $309(53.5)$ \\
SIDA & $882(70.3)$ & - & - \\
Acc. tráfico & $39(3.1)$ & $131(15)$ & $86(14.9)$ \\
Suicidio & $28(2.2)$ & $81(9.2)$ & $42(7.3)$ \\
Enf. hepática & $50(4)$ & $34(3.9)$ & $13(2.2)$ \\
Otras causas & $36(2.9)$ & $62(7.1)$ & $38(6.6)$ \\
Sin datos & $35(2.8)$ & $60(6.8)$ & $90(15.6)$ \\
Total $(\%)$ & $1254(46.3)^{*}$ & $876(32.3)^{*}$ & $578(21.4)^{*}$ \\
${ }^{*}=$ porcentaje de la fila & & & \\
\hline
\end{tabular}

Tabla 5. Edad, años de dependencia y duración del tratamiento por el Ser.T.

\begin{tabular}{|c|c|c|c|}
\hline & M (SD) & $F(S D)$ & Totale \\
\hline $\begin{array}{l}\text { Sobredosis } \\
\text { Edad }[876+125=1001] \\
\text { Años de dependencia }[769+112=881] \\
\text { Años de tratamiento }[865+123=988]\end{array}$ & $\begin{array}{c}29.9(5.8) \\
10.1(5,4) \\
5.5(4.1)\end{array}$ & $\begin{array}{c}30(6.6)^{\dagger} \\
10.5(5.1)^{\dagger} \\
5.8(4.3)^{\dagger}\end{array}$ & $\begin{array}{l}29.9(5.9) \\
10.1(5.4) \\
5.6(4.1)\end{array}$ \\
\hline $\begin{array}{l}\text { SIDA } \\
\text { Edad }[702+180=882] \\
\text { Años de dependencia }[652+171=823] \\
\text { Años de tratamiento }[693+177=870]\end{array}$ & $\begin{array}{c}33.5(4.9) \\
14.4(4.3) \\
9(4.1)\end{array}$ & $\begin{array}{c}33.1(5.4)^{\dagger} \\
13.8(3.9)^{+} \\
8.8(4)^{+}\end{array}$ & $\begin{array}{l}33.4(5) \\
14.3(4.2) \\
9(4.1)\end{array}$ \\
\hline $\begin{array}{l}\text { Accidentes tráfico } \\
\text { Edad }[221+35=256] \\
\text { Años de dependencia }[193+34=227] \\
\text { Años de tratamiento }[219+35=254]\end{array}$ & $\begin{array}{l}28.7(5.4) \\
8.7(4.7) \\
4.6(3.6)\end{array}$ & $\begin{array}{c}26.1(4.7)^{*} \\
7.2(4.2)^{\dagger} \\
3.8(3.1)^{\dagger}\end{array}$ & $\begin{array}{l}28.3(5.4) \\
8.5(4.7) \\
4.5(3.5)\end{array}$ \\
\hline $\begin{array}{l}\text { Suicidio } \\
\text { Edad }[129+22=151] \\
\text { Años de dependencia }[122+21=143] \\
\text { Años de tratamiento }[129+22=151]\end{array}$ & $\begin{array}{l}30.5(7.2) \\
9.1(5.4) \\
5.5(4.2)\end{array}$ & $\begin{array}{l}29(5.7)^{\dagger} \\
9.7(4.7)^{\dagger} \\
6.6(3.6)^{\dagger}\end{array}$ & $\begin{array}{l}30.3(7) \\
9.2(5.3) \\
5.7(4.1)\end{array}$ \\
\hline $\begin{array}{l}\text { Hepatopatia } \\
\text { Età }[83+14=97] \\
\text { Años de dependencia }[77+13=90] \\
\text { Años de tratamiento }[81+13=94]\end{array}$ & $\begin{array}{c}33.4(5.9) \\
14(5.5) \\
8(4.8)\end{array}$ & $\begin{array}{c}34.2(6)^{\dagger} \\
13(4.3)^{+} \\
10(3)^{+}\end{array}$ & $\begin{array}{c}33.5(5.9) \\
13.9(5.3) \\
8.3(4.6)\end{array}$ \\
\hline $\begin{array}{l}\text { Otras causas } \\
\text { Età }[106+30=136] \\
\text { Años de dependencia }[96+27=123] \\
\text { Años de tratamiento }[104+30=134]\end{array}$ & $\begin{array}{l}33.4(7.3) \\
12.5(5.6) \\
6.7(4.7)\end{array}$ & $\begin{array}{l}31.4(6.8)^{\dagger} \\
12.1(5.5)^{\dagger} \\
7.9(5.6)^{\dagger}\end{array}$ & $\begin{array}{c}33(7.2) \\
12.4(5.5) \\
7(4.9)\end{array}$ \\
\hline $\begin{array}{l}\text { Sin datos } \\
\text { Età }[153+32=185] \\
\text { Años de dependencia }[123+22=145] \\
\text { Años de tratamiento }[147+30=177]\end{array}$ & $\begin{array}{l}31.1(6.1) \\
11.6(5.5) \\
6.7(4.5)\end{array}$ & $\begin{array}{c}30.8(6.5)^{\dagger} \\
11(5.7)^{+} \\
7.1(4.7)^{\dagger}\end{array}$ & $\begin{array}{l}31(6.2) \\
11.5(5.5) \\
6.7(4.5)\end{array}$ \\
\hline $\begin{array}{l}\text { Total } \\
\text { Età }[2270+438=2708] \\
\text { Años de dependencia }[2032+400=2432] \\
\text { Años de tratamiento }[2238+430=2668]\end{array}$ & $\begin{array}{c}31.3(6) \\
11.6(5.5) \\
6.8(4.4)\end{array}$ & $\begin{array}{c}31.2(6.3)^{+} \\
11.8(5)^{+} \\
7.3(4.5)^{*}\end{array}$ & $\begin{array}{c}31.3(6) \\
11.7(5.4) \\
6.8(4.5)\end{array}$ \\
\hline
\end{tabular}


ADVP fallecidos por SIDA y enfermedades hepáticas habían tenido un periodo de acogida más largo ( 9 y 8.3 años respectivamente) $(p<0.001)$, mientras que el de los sujetos fallecidos por accidente de tráfico era más corto (4.5 años), seguidos de los ADVP muertos por sobredosis y suicidio (5.6 y 5.7 años respectivamente) $(p<0.001)$.

Edad media de muerte por año de observación. La edad media de muerte por año de observación aparece en la Fig. 2. La edad media fue aumentando desde los 26-27 años a mediados de los años 80, a los 34.1 años en 1998 ( $p<0.001$ ).

Tasa de mortalidad de los ADVP vs la del conjunto de la población. En la Tabla 6 se compara la tasa de mortalidad de nuestro grupo de ADVP con la del conjunto de la población en 1991, calculada en base al último censo. En las columnas 2 y 6 se indica el número de ADVP muertos en 1991 y el número de muertes estimado.

En los hombres, la tasa de mortalidad de los ADVP de las tres categorías de edad comprendidas entre 25 y 39 años resultó aproximadamente tres veces más alta que la tasa de mortalidad de la franja de edad comprendida entre los 20-24 y entre las dos franjas de edad comprendidas entre los 40-59; los resultados de las mujeres son similares, aunque con menos diferencias.

La tasa de mortalidad de los ADVP que acudieron a los 36 servicios analizados era significativamente más alta que la del conjunto de la población de la misma edad (13 veces, 95\% Cl, 11.3-14.6) ( $p<0.001)$. Considerando el intervalo de confianza de la SMR entre los hombres, la mortalidad de los ADVP en las franjas de edad de los 20-24 y 40-59 años resulta similar, y significativamente más baja que la SMR de los ADVP de
25-39 años. En las mujeres las diferencias de la SMR no son significativas, probablemente porque el intervalo de confianza es grande debido a la escasez de mujeres en la población de drogodependientes. Si se comparan los sexos, los hombres presentan una SMR de $8.8(95 \% \mathrm{Cl}, 7.7-10.2)$, significativamente más baja que la de las mujeres, que tienen una SMR de 21.7 (95\% Cl, 15.7-29.2).

Por último, en el año del censo, el porcentaje de muertes causadas por la droga en el grupo de edad comprendido entre los 15-34 del total de la población (195 muertos entre los ADVP/1241 muertes en el conjunto de la población, Tabla 6), resultó ser del 16\%, $17 \%$ en los hombres y $12 \%$ en las mujeres respectivamente.

\section{DISCUSIÓN}

Confirmando estudios nacionales ${ }^{2,5}$ e internacionales $^{7,22-24}$ la SMR de los ADVP acogidos en nuestros Servicios resultó ser 13 veces más elevada que la del conjunto de la población del mismo grupo de edad. Al comparar los datos que obtuvimos sobre la tasa de mortalidad con los de otros estudios del norte de Italia se comprobó que eran menores que los de Bolonia (18 veces) y Milán (20 veces)., ${ }^{3,5}$

Por sexos, la diferencia entre la SMR de los ADVP y del conjunto de la población (8.85 en los hombres, 21.71 en las mujeres) no debe atribuirse al aumento de la tasa de mortalidad de las ADVP mujeres. El Indirectly Standardised Rate de los hombres y de las mujeres es parecido (2.56 2.60 respectivamente). La diferencia se debe a que la tasa de mortalidad de las

Figura 2. Distribución de la edad media de las muertes desde 1985 al 1998

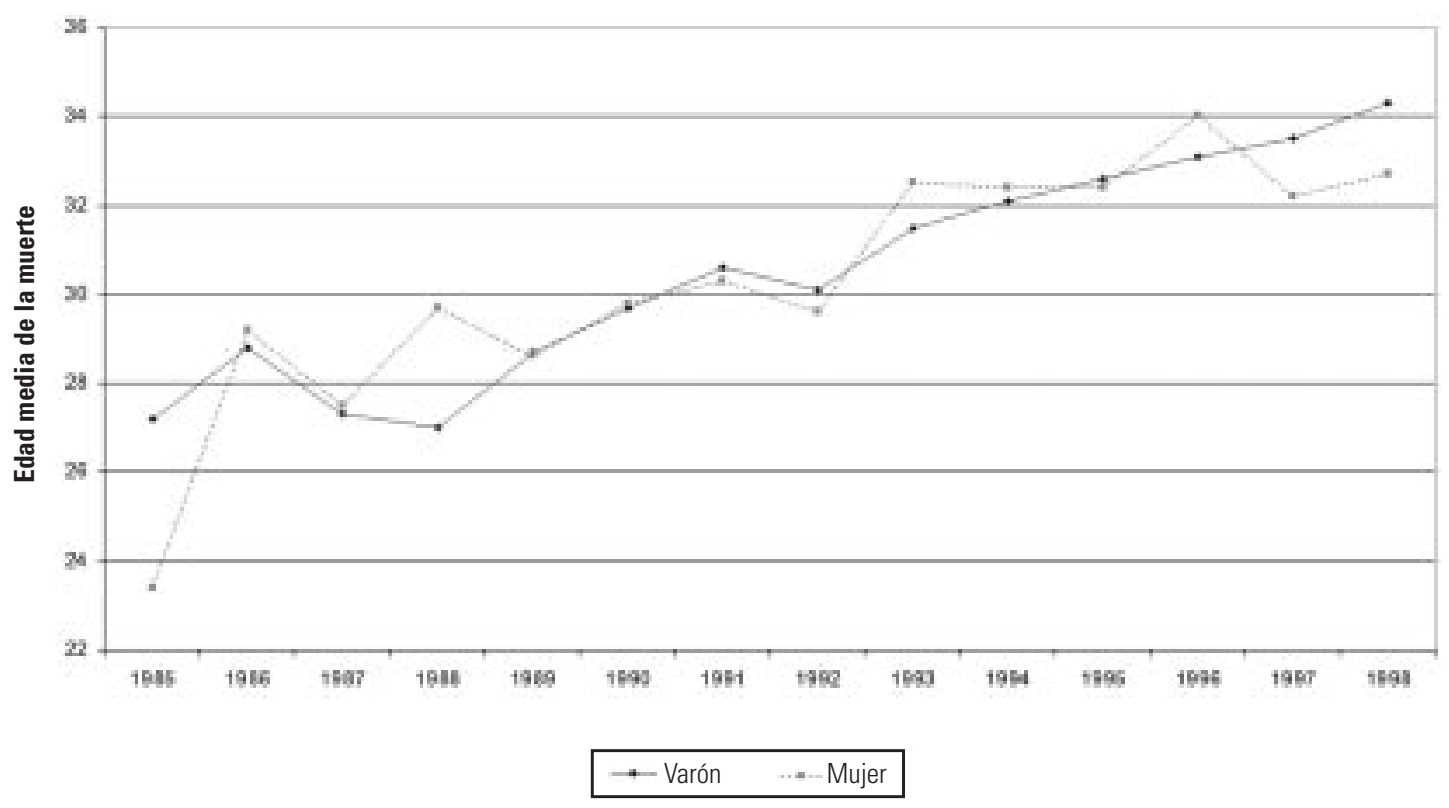


Tabla 6. Comparación entre entre la Standardized Mortality Ratio (SMR) de los 2.708 ADVP y la SMR de la Población General (GP) en 1991 después de corregir por edad y sexo

\begin{tabular}{|c|c|c|c|c|c|c|c|c|}
\hline Edad & $\begin{array}{l}N^{\circ} \text {. muertos } \\
\text { entre los ADVP }\end{array}$ & $\begin{array}{l}N^{\circ} . \text { de } \\
\text { ADVP }\end{array}$ & $\begin{array}{l}\mathrm{N}^{\circ} \text {. de muertos } \\
\text { entre la PG }\end{array}$ & $\begin{array}{l}N^{\circ} . \text { de } \\
\text { la PG }\end{array}$ & $\begin{array}{l}\mathrm{N}^{\circ} \text {. de muertos } \\
\text { esperados }\end{array}$ & $\begin{array}{l}\text { SMR } \\
\text { (O/E) }\end{array}$ & $95 \% \mathrm{Cl}$ & $\mathbf{P}$ \\
\hline \multicolumn{9}{|c|}{ Varones } \\
\hline 15-19 & 4 & 313 & 180 & 182,015 & 0.31 & 12.9 & $3.52-33.03$ & 0.001 \\
\hline 20-24 & 21 & 1983 & 253 & 206,961 & 2.38 & 8.82 & $5.46-13.49$ & 0.001 \\
\hline $25-29$ & 77 & 3085 & 253 & 218,731 & 3.7 & 20.81 & $16.4-26.0$ & 0.001 \\
\hline 30-34 & 57 & 1588 & 256 & 190,633 & 2.06 & 27.67 & $21-35.8$ & 0.001 \\
\hline 35-39 & 31 & 575 & 270 & 171,824 & 0.92 & 33.69 & $22.9-47.8$ & 0.001 \\
\hline $40-59$ & 10 & 284 & 3351 & 629,733 & 1.35 & 7.41 & $3.6-13.6$ & 0.001 \\
\hline $15-59$ & $\Sigma=200$ & 7796 & 4563 & $1,599,897$ & 22.61 & 8.85 & 7.7-10.2 & 0.001 \\
\hline \multicolumn{9}{|c|}{ Mujeres } \\
\hline 15-19 & 1 & 90 & 49 & 174,281 & 0.027 & 37.04 & $1.1-206.3$ & 0.013 \\
\hline $20-24$ & 4 & 428 & 70 & 197,072 & 0.17 & 23.6 & $6.4-60.2$ & 0.001 \\
\hline $25-29$ & 16 & 609 & 77 & 209,005 & 0.24 & 66.7 & $38.1-108.2$ & 0.001 \\
\hline 30-34 & 15 & 333 & 103 & 182,043 & 0.2 & 75 & $42-123.7$ & 0.001 \\
\hline 35-39 & 6 & 121 & 114 & 166,343 & 0.08 & 75 & $27.5-163.2$ & 0.001 \\
\hline $40-59$ & 1 & 72 & 1566 & 775,683 & 0.14 & 7.14 & $0.21-39.8$ & 0.070 \\
\hline $15-59$ & $\Sigma=43$ & 1653 & 1979 & $1,704,427$ & 1.98 & 21.71 & $15.7-29.2$ & 0.001 \\
\hline \multicolumn{9}{|c|}{ Varones + Mujeres } \\
\hline $15-59$ & $\Sigma=243$ & 9451 & 6542 & $3,304,374$ & 18.90 & 12.85 & $11.3-14.6$ & 0.001 \\
\hline
\end{tabular}

mujeres en el conjunto de la población es más baja (0.12:0.29). Este resultado, que confirma un estudio reciente, ${ }^{25}$ revela la importancia del riesgo de muerte de las mujeres ADVP: por ello los trabajadores de los servicios deben concederles una atención especial, tal y como ha puntualizado Frischer. ${ }^{24}$

El porcentaje (16\%) de muertes atribuibles a la drogodependencia en la población general, que coincide con el de otra investigación italiana ${ }^{26}$ es sorprendente si se considera que en la zona en la que se realizó el estudio el número de ADVP pertenecientes al mismo grupo de edad era aproximadamente el $1 \%$ de la población.

El continuo aumento de muertes entre los ADVP de la zona estudiada, especialmente después de 1988, coincide con la tendencia nacional. ${ }^{27}$ El significativo descenso de las muertes por SIDA que se aprecia en los dos últimos años examinados se debe probablemente a los nuevos protocolos terapéuticos antirretrovirales $^{28}$ que en Italia siguen siendo prácticamente gratuitos para todo tipo de paciente. En un estudio realizado en Nueva York ${ }^{29}$ se ha revelado la misma tendencia en todas las categorías con riesgo de transmisión del VIH y en todos los grupos raciales. El descenso de la mortalidad estuvo precedido de una caída en la incidencia de casos de VIH/SIDA entre los ADVP. ${ }^{30}$ Por ello la prevención y el tratamiento de las infecciones de los ADVP seropositivos que no sean SIDA, especialmente la hepatitis $C$ y el cáncer, adquirirá cada vez más importancia. ${ }^{31}$

El hecho de que la sobredosis siga siendo una causa de muerte tan importante requiere nuevos estudios. Debe subrayarse el hecho de que a pesar de los importantes cambios introducidos en la legislación y de las líneas seguidas en los tratamientos de metadona, las muertes por sobredosis han permanecido constantes durante muchos años. La restricción de la dosis de metadona se suprimió en 1993, por lo que coincidiendo con otros autores ${ }^{32,33}$ nuestros resultados sugieren que la influencia de las políticas gubernamentales sobre la mortalidad de los ADVP es limitada. Las campañas preventivas deberían enfatizar el peligro de la sobredosis junto al del SIDA y la hepatitis.

Los fracasos de los tratamientos de mantenimiento con metadona constituyen además un importante factor de riesgo de sobredosis. ${ }^{34,35}$ Según nuestra experiencia, esta consideración debe extenderse a tratamientos de rehabilitación como los programas con naltrexona, las comunidades terapéuticas y los tratamientos psicoterapéuticos. Otros de los factores de riesgo de sobredosis más importantes son la variación del porcentaje de heroína en la droga de la calle, más que el aumento de la pureza en sí misma; el uso concurrente de otras drogas, y especialmente del 
alcohol, $^{33,36-38}$; la disponibilidad de droga; la disminución en la tolerancia a la heroína ${ }^{39} \mathrm{y}$, como ha sido advertido en ocasiones, la edad juvenil y la brevedad del periodo de tratamiento en los Ser.T. Para intentar reducir eficazmente el riesgo de muerte por sobredosis deberían estudiarse vías para poder prevenir y, por lo tanto, reducir los drop-out de los tratamientos. ${ }^{34}$ Lógicamente, los heroinómanos deben ser alertados sobre el peligro potencial que supone el consumo simultáneo de otras drogas sobre los efectos de la heroína, y sobre todo sobre el aumento de la vulnerabilidad de los bebedores de alcohol. ${ }^{36}$ Las campañas dirigidas al aumento de la concienciación del drogodependiente también deberían insistir en que es más seguro inyectarse heroína en compañía de otras personas y que en los casos de sobredosis es muy importante llamar a una ambulancia. También debería contemplarse la posibilidad de realizar periódicamente análisis de la heroína de la calle, ${ }^{38}$ de distribuir naloxona $^{37}$ usando preferiblemente jeringuillas ya prepara$\mathrm{das}^{40}$, y por último, insistir sobre el hecho de que el uso de heroína ilegal y los comportamientos criminales disminuyen en los casos en los que ha sido posible recetar heroína. ${ }^{41}$

Además de todo esto, es muy probable que el número de muertes por sobredosis esté subestimado porque haya casos en los que los fallecimientos se certifiquen como muertes por causas naturales para evitar la autopsia o el registro como muerte debida a abuso de drogas, un dato que ha sido señalado por muchos autores. ${ }^{42}$

El elevado porcentaje de muertes causadas por accidentes de tráfico, analizada en un trabajo que realizamos anteriormente ${ }^{13}$, se confirma, aunque con una leve pérdida (del 11 al 9.5\%). Este resultado sin embargo no se puede comparar con otros estudios que consideran los accidentes de tráfico dentro de la categoría de muertes violentas. ${ }^{2,11}$ El suicidio incide en un $5.6 \%$ de las muertes, dato similar a los que aparecen en otras investigaciones ${ }^{2,5}$ pero más bajo que los datos de estudios realizados en el norte de Europa. ${ }^{43,44}$

La mortalidad originada por hepatopatías es sorprendentemente baja, aunque en la zona estudiada, en 1985, la prevalencia media del VHC estuviese cerca del $70 \%$ y la del VHB fuese del $40 \%$, reduciéndose después al $20 \%$ en 1998. ${ }^{45,46}$ Además, existen estudios anteriores que aportan porcentajes sólo ligeramente más altos que los nuestros. 5,47 Por otra parte, también se ha podido confirmar posteriormente que la hepatopatía provocada por los virus C y D son más agresivas en individuos positivos al VIH, acelerando la insuficiencia hepática. ${ }^{48,49}$

Respecto a las muertes por patologías infecciosas, el reducidísimo número de muertes por endocarditis también es sorprendente. No se ha documentado ningún caso de TBC entre las causas de muerte, a pesar de la existencia de estudios previos que han subrayado el aumento de TBC entre los ADVP. ${ }^{50,51}$

Es más, existe un estudio italiano que ha demostrado que en los ADVP positivos al VIH sólo existe un aumento modesto en el riesgo de TBC. ${ }^{52}$

Respecto a la tasa de seropositividad al $\mathrm{VIH}$, el único dato significativo es que, si exceptuamos a los pacientes muertos por SIDA, la prevalencia es mayor en los individuos fallecidos por hepatopatías, dato que coincide con los resultados de estudios precedentes. ${ }^{3,18,53}$ El estudio no ha revelado que el porcentaje de seropositividad al VIH sea más alto entre los ADVP muertos por sobredosis o suicidio. En este aspecto, algunos estudios confirman nuestros datos, 5,6,18,54 al contrario que otros..$^{1,455}$ Además de ello, la notificación de seropositividad al VIH a los ADVP no parece suponer un imprevisto y real aumento de las muertes por sobredosis-suicidio. ${ }^{18}$ Finalmente, el relativamente alto número de ADVP con una situación serológica al VIH desconocida se debe al hecho de que en Italia el test es voluntario, y más de un paciente prefiere hacérselo en centros que no sean Ser.T. en los que es más fácil mantener el anonimato.

La edad media de muerte ha subido de los 26-27 años de mediados de los años 80 a los 34 años en 1998. Kringsholm ${ }^{56}$ atribuye el visible aumento de la edad media de la muerte en su grupo de ADVP a una disminución del número de nuevos individuos adictos al consumo de drogas. Esta conclusión no puede aplicarse a los ADVP de nuestra zona, en la que, como muestran los datos del Ministerio de Sanidad, ${ }^{57}$ el aumento de ADVP que solicitan tratamiento en los Ser.T. es continuo. Lo más probable es que ese fenómeno se deba a los cambios de los hábitos y el estilo de vida de los ADVP, que actualmente están mucho más integrados en la sociedad, viven en casa o en familia, tienen una ocupación normal y cuidan de su salud mucho más que en el pasado.

Nuestro trabajo es el más extenso que se ha publicado en Europa sobre la mortalidad relacionada con el consumo de heroína. El resultado más significativo ha sido la marcadísima reducción de las muertes causadas por el SIDA a partir de 1997 en adelante, el número de las muertes debidas a sobredosis que representa la principal causa de muerte y la tasa de mortalidad entre los ADVP, que ha demostrado ser 13 veces mayor que la del conjunto de la población de la misma edad. La edad media de muerte aumenta continuamente año tras año. La prevalencia del VIH no supone un factor significativo entre las causas de suicidio o las muertes por sobredosis.

Las consecuencias prácticas de esta investigación, para aquellos que trabajan en el mundo de la drogodependencia, son que debería prestarse una atención mucho mayor a las mujeres toxicómanas, porque están sujetas a un riesgo de muerte mayor. También deberían 
enfatizarse mucho más los peligros de la sobredosis entre los ADVP, así como la importancia de no abandonar los tratamientos, sean del tipo que sean, porque está demostrado que el riesgo de sobredosis aumenta inmediatamente después del drop-out. Este último problema podría resolverse estimulando la cooperación entre los Ser.T., los médicos de base, las comunidades terapéuticas, las instituciones penales, etc.

Los accidentes de tráfico se han confirmado como una importante causa de muerte; los psicofármacos deberían suministrarse con una extremada precaución, porque frecuentemente se asocian al abuso de alcohol, lo que representa una importante causa colateral de accidentes de carretera.

La disminución de las muertes por SIDA exige una paralela intensificación de los esfuerzos dirigidos a la prevención de la hepatitis $\mathrm{C}$, así como renovar los esfuerzos destinados a curarla.

Las medidas que se tomen en el campo de las drogodependencias, sea en el terreno de la política como en el de la investigación y de la formación, deben tener en cuenta el dramáticamente alto porcentaje de muertos por consumo de heroína.

\section{AGRADECIMIENTOS}

Han colaborado en el presente estudio:

F. Arisi, E. Baggio, V. Balestra, D. Barbiero, G. Bellio, M. Bortolomasi, C. Bossi, R. Bressan, R. Bricolo, D. Cantiero, C. Capra, L. Carraro, R. Casari, F. Celeghin, R. Ceravolo, G. Cicciù, P. Civitelli, F. Corso, D. Danieli, D. Dariol, E. Dellantonio, G. Didonè, B. Durano, M. Faccini, S. Ferrari, D. Ferremi, G. Forza, M. Frezza, G. Ghirardello, MA. Giacomin, R. Giusto, F. Guidoboni, E. Levarda, A. Lo Russo, R. Lovaste, G. Mantovani, E. Manzato, M. Mazzo, V. Mecenero, PG. Nardi, A. Padovani, B. Pajusco, A. Pani, A. Parolin, P. Pili, S. Polli, M. Residori, A. Rovea, R. Sabbion, C. Saccardi, M. Sarti, L. Sartori, M. Scaggiante, A. Scandolari, F. Schifano, C. Smacchia, K. Sonnerer, I. Spilimbergo, C. Stimolo, P. Tito, V. Vasi, A. Vendramin, F. Viviani, M. Von Solder, F. Zuin.

\section{BIBLIOGRAFIA}

(1) Zaccarelli, M., Gattari, P., Rezza, G., Conti, S., Spizzichino, L., Vlahov, D., Ippolito, G., Lelli, V. \& Valenzi, C. (1994) Impact of HIV infection on non-AIDS mortality among Italian injecting drug users, AIDS, 8, 345-350.

(2) Perucci, C.A., Davoli, M., Rapiti, E., Forastiere, F. \& Abeni, D.D. (1991) Mortality of intravenous drug users in Rome: a cohort study, American Journal of Public Health, 81, 1307-1310.
(3) Goedert, J.J., Pizza, G., Gritti, F.M., Costigliola, P., Boschini, A., Bini, A., Lazzari, C. \& Palareti, A. (1995) Mortality among drug users in the AIDS era, International Journal of Epidemiology, 24, 1204-1210.

(4) Eskild, A., Magnus, P., Samuelsen, S.O., Sohlberg, C. \& Kittelsen, P. (1993) Differences in mortality rates and causes of death between HIV positive and HIV negative intravenous drug users, International Journal of Epidemiology, 22, 315-320.

(5) Galli, M. \& Musicco, M., for the COMCAT Study Group (1994) Mortality of intravenous drug users living in Milan, Italy: role of HIV-1 infection, AIDS, 8, 1457-1463.

(6) Stoneburner, R.L., Des Jarlais, D.C., Benerza, D., Gorelkin, L., Sotheran, J.L., Friedman, S.R., Schultz, S., Marmor, M., Mildvan, D. \& Maslansky, R. (1988) A larger spectrum of severe HIV-1-related disease in intravenous drug users in New York City, Science, 242, 916-919.

(7) Selwyn, P.A., Hartel, D., Wasserman, W. \& Drucker, E. (1989) Impact of the AIDS epidemic on morbidity and mortality among intravenous drug users in a New York City methadone maintenance program, American Journal of Public Health, 79, 1358-1362.

(8) Orti, R.M., Domingo-Salvany, A., Munoz, A., Mactarlane, D., Suelves, J.M. \& Antò, J.M. (1996) Mortality trends in a cohort of opiate addicts, Catalonia, Spain, International Journal of Epidemiology, 25, 545-553.

(9) Vaillant GE. (1973) A 20 year follow up of New York narcotic addicts, Archives in General Psychiatry, 29, 237-241.

(10) Thorsen, K. \& Haastrup, S. (1975) Follow up of 97 young non-psychotic male opiate abusers: relationship between achiving abstinence, age, and duration of abuse, Acta Psychiatrica Scandinavica, 51, 289-296.

(11) Barr, H.L., Antes, D., Ottenberg, D.J. \& Rosen, A. (1984) Mortality of treated alcoholics and drug addicts; the benefits of abstinence, Journal of Studies on Alcohol, 45, 440-452.

(12) Bucknall, A.B.V. \& Robertson, J.R. (1986) Deaths of heroin users in a general practice population, Journal of Royal College of General Practitioner, 36,120-122.

(13) Mezzelani, P., Quaglio, G.L., Venturini, L., Lugoboni, F., G.I.C.S., Friedman, S.R. \& Des Jarlais D.C. (1998) A multicentre study on the causes of death among Italian injecting drug users. AIDS has overtaken overdose as the principal cause of death, AIDS Care, 10, 61-67.

(14) Mezzelani, P., Venturini, L., Turrina, G., Lugoboni, F. \& Des Jarlais, D.C. (1991) High compliance with a hepatitis B virus vaccination program among intravenous drug users, Journal of Infectious Diseases, 163,923

(15) Popolazione residente per sesso, età, regioni 1999. Istituto Italiano di Statistica (ISTAT). Roma 2000. [Resident population by sex, age, in Regions-Italy 1999. National Institute of Statistics. Roma 2000].

(16) Indagine trimestrale sulle forze di lavoro 1999. Istituto Italiano di Statistica (ISTAT). Roma 2000. [ Trimestral report on working class data1999. National Institute of Statistics. Roma 2000]. 
(17) Conti economici regionali 1996-1998. Istituto Italiano di Statistica (ISTAT). Roma 1998. [Regional financial budget 1996-1998. National Institute of Statistics. Roma 1998].

(18) van Haastrecht, H.J.A., Mientjes, G.H.C., van den Hoek, J.A.R. \& Coutinho, R.A. (1994) Death from suicide and overdose among drug injectors after disclosure of first HIV test result, AIDS, 8, 1721-5.

(19) Censimento Demografico Nazionale 1991; un modello di analisi per le realtà locali. (1996) Regione Veneto. Dipartimento per la statistica e l'informatica. [National Population Census 1991; an analysis model for local districts. Veneto Region. Department of applied statistics].

(20) Tredicesimo censimento della popolazione. 20 Ottobre 1991. Istituto Nazionale di Statistica (ISTAT). Roma 1993. [Thirteenth population census. 20 October 1991. National Institute of Statistics. Roma 1993].

(21) Breslow, M. \& Day, N. (1986) Rates and rate standardization. Statistical methods in cancer research. Lyon, France: International Agency for Research on Cancer (IARC), 48-79.

(22) Haastrup, S. \& Jepsen, P.W. (1984) Seven year follow-up of 300 young drug abusers, Acta Psychiatrica Scandinavica, 70, 503-09.

(23) Joe, G.W. \& Simpson, D.D. (1987) Mortality rate among opiate addicts in a longitudinal study, American Journal of Public Health, 77, 347-348.

(24) Frisher, M., Bloor, M., Goldberg, D., Clark, J., Green, S. \& McKeganey, N. (1993) Mortality among injecting drug users: a critical reappraisal, Journal of Epidemiology and Community Health, 47, 59-63.

(25) Davoli, M., Perucci, C., Rapiti, E., Barbagli, A.M., D'Ippoliti, D., Forastiere, F. \& Abeni, D.D. (1997) A persistent rise in mortality among injection drug users in Rome, 1980 through 1992, American Journal of Public Health, 87, 851-853.

(26) Perucci, C.A., Forastiere, F., Rapiti, E., Davoli, M. \& Abeni, D.D. (1992) The impact of intravenous drug use on mortality of young adults in Rome, Italy, British Journal of Addiction, 81, 1247-1249.

(27) Relazione annuale della Direzione Centrale per i Servizi Antidroga. (1998), Ministero dell'Interno, Roma. [Annual Report of Head Office of Public Centres for Drug Users 1998. Home Office, Roma, 1998, 130-132].

(28) Hogg, R.S., O'Shaughnessy, M.V., Gataric, N., Yip, B., Craib, K., Schechter, M.T. \& Montaner JS (1997) Decline in deaths from AIDS due to new antiretrovirals, Lancet, 349:1294.

(29) Chiasson, M.A., Berenson, L., Li, W., Schwartz, S., Singh, T., Forlenza, S., Mojica, B.A. \& Hamburg, M.A. (1999) Declining HIV/AIDS mortality in New York City, Journal of Acquired Immune Deficiency Syndromes, 21:59-64.

(30) Baggio, E., Bossi, C., Bressan, R., Ceravolo, R., Durano, B., Giacomini, M.A., Lugoboni, F., Manzato, E., Rossetto, L., Saccardi, C., \& Stimolo C. (1995) L'infezione da virus dell'immunodeficienza umana tra i tossicodipendenti da eroina: studio epidemiologico in 10 Servizi per le Tossicodipendenze del Veneto. 1985-1992 [HIV infection among heroin drug users: epidemiological study in 10 Public Health Authority Centres for Drug Users in Veneto Region.1985-1992]. Giornale Italiano dell'AIDS, 6, 154156.

(31) Prins, M., Sabin, C.A., Lee, C.A., Devereux, H., \& Coutinho, R.A. (2000) Pre-AIDS mortality and its association with HIV disease progression in haemophilic men, injecting drug users and homosexual men, AIDS, 14, 1829-837.

(32) Ghodse, A.H., Sheehan, M., Taylor, C. \& Edwards, G. (1985) Deaths of drug addicts in the United Kingdom 1967, British Medical Journal, 290, 425-428.

(33) Hulse, G.K., English, D.R., Milne, E. \& Holman C.D.J. (1999) The quantification of mortality resulting from the regular use of illicit opiates, Addiction, 94, 221-229.

(34) Davoli, M., Perucci, C., Forastiere, F., Doyle, P., Rapiti, E., Zaccarelli, M. \& Abeni, D.D. (1993) Risk factors for overdose mortality: a case-control study within a cohort of intravenous drug users, International Journal of Epidemiology, 22, 273-277.

(35) Gronbladh, L., Ohlund, L.S. \& Gunne, L.M. (1990) Mortality in heroin addiction: impact of methadone treatment, Acta Psychiatrica Scandinavica, 82, 223-227.

(36) Ruttenber, A.J., Kalter, H.D. \& Santinga, P. (1990) The role of ethanol abuse in the etiology of heroin-related death, Journal of Forensic Science, 35, 891-900.

(37) Zador, D., Sunjic, S. \& Darke, S. (1996) Heroin-related deaths in New South Wales, 1992: toxicological findings and circumstances, Medical Journal of Australia, 164, 204-207.

(38) Darke, S., Sunjic, S., Zador, D. \& Prolov, T. (1997) A comparison of blood toxicology of heroin-related deaths and current heroin users in Sidney, Australia, Drug and Alcohol Dependence, 47, 45-53.

(39) Seaman, S.R., Brettle, R.P, \& Gore, M. (1998) Mortality from overdose among injecting drug users recently released from prison: database linkage study, British Medical Journal, 316, 426-428.

(40) Strang, J., Darke, S., Hall, W., Farrell, M., \& Ali, R. (1996) Heroin overdose: the case for take-home naloxone, British Medical Journal, 312, 1435-436.

(41) Bammer, G., Dobler-Mikola, A., Fleming, P.M., Strang, J. \& Uchtenhagen, A. (1999) The heroin prescribing debate: integrating science and politics, Science, 284, 1277-8.

(42) De La Fuente, L., Barrio, G., Vicente, J., Bravo, M.J. \& Santacreu, J. (1995) The impact of drug-related deaths on mortality among young adults in Madrid, American Journal of Public Health, 85, 102-105.

(43) Haastrup, S. \& Jepsen, P.W. (1988) Eleven-year followup of 300 young opioid addicts, Acta Psychiatrica Scandinavica, 77, 22-26.

(44) Fugelstad, A., Annell, A., Rajs, J. \& Agren, G. (1997) Mortality and causes and manner of death among drug addicts in Stockholm during the period 1981-1992, Acta Psychiatrica Scandinavica, 96, 169-175.

(45) Lugoboni, F., Mezzelani, P., Venturini, L., Fibbia, G.C. \& Des Jarlais D.C. (1992) An HBV vaccination program for street injecting drug users: implications for testing an 
HIV vaccine. VIII International Conference on AIDS, Amsterdam [abstract PoC 4796].

(46) Mezzelani, P., Venturini, L., Fibbia, G.C., Perdonà, G.P., Lugoboni, F., Faccini, M., Bossi, C., Saccardi, C. \& Des Jarlais D.C. (1992) Parallel decline in both HIV and HBV seroprevalence following AIDS prevention programs among injecting drug users in North-East Italy. VIII International Conference on AIDS, Amsterdam [abstract PoC 4797].

(47) Soriano, V., Garcia-Samaniego, J., Valencia, E., Rodriguez-Rosado, R., Munoz, F. \& Gonzalez-Lahoz, J. (1999) Impact of chronic liver disease due to hepatitis viruses as cause of hospital admission and death in HIV-infected drug users, European Journal of Epidemiology, 15, 1-4.

(48) Telfer, P., Sabin, C., Devereux, H., Scott, F. \& Dusheiko, G. (1994) The progression of HCV-associated liver disease in a cohort of haemophilia patients, British Journal of Haematology, 87, 555-561.

(49) De Pouplana, M., Soriano, V., Garcia-Samaniego, J., Enriquez, A., Munoz, F., \& Gonzalez-Lahoz, J. (1995) More severe course of Delta hepatitis in HIV-infected patients, Genitourinary Medicine, 71, 132-133.

(50) Haas, D.W. \& Des Prez, R.M. (1994) Tuberculosis and acquired immunodeficiency syndrome: a historical perspective on recent developments, American Journal of Medicine, 96, 439-450.

(51) Braun, M.M., Cotè, T.R. \& Rabkin, C.S. (1993) Trend in death with tuberculosis during the AIDS era, Journal of the American Medical Association, 269, 2865-2868.

(52) Antonucci, G., Armignacco, O. \& Girardi, E. (1990) Tubercolosi e infezione da HIV: incidenza della tubercolosi in soggetti con infezione da HIV e prevalenza di sieropositività per HIV-Ab in pazienti con tubercolosi. [Tuberculosis and HIV infection: incidence of tuberculosis in HIV positive subjects and prevalence of HIV seropositivity in patients with tuberculosis] In: Second Research Project on AIDS, 1989. ISTISAN Reports 90/10:133. Edizioni G.B. Rossi. [G.B.Rossi Publisher].

(53) van Ameijden, E.J.C., Vlahov, D., van den Hoek, J.A.R., Flynn, C. \& Coutinho, R.A. (1996) Pre-AIDS mortality and morbidity among injection drug users in cohort studies in Amsterdam and Baltimore, XI International Conference on AIDS, Vancouver [abstract ThC220].

(54) Soellner, R., Castrup, U., Hechmann, W., Teschke, F. \& Vorwold M. (1992) Does an HIV-infection increase the probability of dying by a drug-overdose?, VIII International Conference on AIDS, Amsterdam [abstract D402]

(55) Prins, M., Aguado, I.H., Brettle, R.P., Robertson, J.R., Broers, B., Carrè, N., Glodberg, D.J., Zangerle, R., Coutinho, R.A. \& van den Hoek, A. (1997) Pre-AIDS mortality from natural causes associated with HIV disease progression: evidence from the European Seroconvert Study among injecting drug users, AIDS, 11, 1747-1756.

(56) Kringsholm, B. (1988) Deaths among drug addicts in Denmark in 1968-1986, Forensic Science International, 38, 139-149.

(57) Rilevazione attività nel settore tossicodipendenze, 1997. Dipartimento della Prevenzione. Ministero della Sanità. [National Report on Addictions, 1997. Department of Prevention. Ministry of Health. Roma 1997]. 
